Kazimierz SZAŁATA

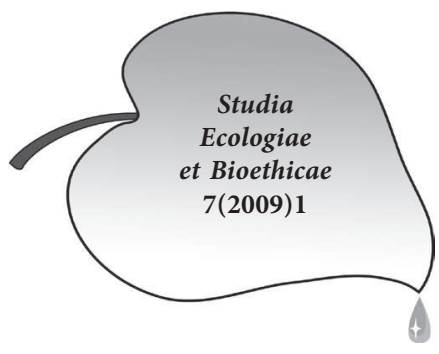

IF WFCh UKSW Warszawa

\title{
Évolution de la conscience morale hippocratique
}

Histoire de l'application du «Serment d'Hippocrate», le plus ancien texte écrit concernant léthique médicale, peut nous permettre de mieux comprendre lévolution de la conscience morale dans notre culture, de l'Antiquité à nos jours.

$\mathrm{Au}$ moins deux raisons nous font réfléchir aujourd'hui sur le patrimoine d'Hippocrate.

- le «Serment d'Hippocrate» est le premier document d'éthique médicale. En tant qu'il relève de la moralité, il reste un excellent exemple pour l'ensemble de l'éthique protégeant la dignité de l'homme.

- après une longue tradition de réflexion morale issue des idées d'Hippocrate, nous sommes les témoins du rejet des valeurs garanties par le «Serment».

Depuis le début du XX'éme siècle, on ne propose plus aux médecins le texte original $\mathrm{du}$ «Serment d'Hippocrate» en le remplaçant par des textes différents presque sans rapport avec le texte original. On affirme même avec un certain mérite que le texte d'Hippocrate est un beau document témoignant d'une certaine conscience morale, mais qui nest qu'un document historique devenu inutile pour nos contemporains. Pour cela nous devons nous inquiéter de ce que notre culture abandonne la tradition la plus digne et les valeurs sur lesquelles a été édifiée notre morale médicale.

\section{Léthique ou la déontologie?}

D’après les recherches historiques, Hippocrate de Cos (460-370 avant J. C.) est né dans une famille de médecins à lépoque d'un fort développement de la philosophie et des sciences ${ }^{1}$. On sait que le texte du «Serment» était connu dans le milieu des Pythagoriciens. Mais selon certains chercheurs, le texte qui est devenu la source de la réflexion morale dans notre culture, est encore beaucoup plus ancien et provient de l'antique culture égyptienne. ${ }^{2}$

1 Hippocrate a sa place dans histoire comme le père de la médecine, qui se détache de la religion et de l'art pour devenir une science.

2 Par exemple Naguibe Rida suggère que l'origine du texte du «Serment d'Hippocrate» provient de l'an 3000 av. J. C. «Refleksje nad etyką lekarską», Warszawa 1992, p 9. 
Il est vrai que depuis le XIX ${ }^{e}$ siècle, à l'époque où l'éthique a été remplacée par la déontologie et les lois positives, il nous est montré de plus en plus souvent un autre exemple, peut-être plus ancien, «le Code d'Hammurabi» ${ }^{3}$. Mais ce code ouvre une autre tradition - celle de la déontologie qui n'est pas la même chose que l'éthique. Il faut savoir distinguer ce qui relève de l'éthique et de la déontologie, donc du droit. Léthique enseigne comment agir selon la vérité de l'homme, comment agir selon un ordre de sagesse, dans la responsabilité et la dignité humaine, alors que la déontologie, donc le droit, nous instruit au sujet des sanctions qui nous attendent si nous tentons de ne pas nous soumettre aux règles inscrites dans le code ou dans la loi. Léthique nous laisse prendre les décisions en toute liberté, tandis que la déontologie et la loi ne nous laissent pas de choix. Il faut dire que ces trois domaines jouent un rôle important dans la vie sociale et dans la vie de chacun d'entre nous. Mais l'un ne peut remplacer l'autre, et surtout la loi ne peut pas remplacer léthique - ce que les milieux positivistes ont tenté de réaliser.

\section{Les principes de l'éthique hippocratique}

Pour mieux redécouvrir la richesse du «Serment d'Hippocrate», il est utile d'analyser d'abord la structure du texte. Au début, nous trouvons une sorte d'invocation indiquant un fondement de la pensée morale. Puis, nous voyons une partie concernant le milieu professionnel, la responsabilité de son développement et sa dignité. Au centre, nous trouvons une forte déclaration sur la protection de la vie humaine comme règle principale et incontestable du «Serment». A la fin du premier code d'éthique médicale, nous avons une déclaration au sujet d'un comportement sur l'un des domaines les plus intimes de l'homme qui exige de notre part une discrétion absolue. Comme clôture, le texte du Serment propose une forte déclaration sur l'honneur.

Nous commençons la lecture à partir d'une invocation qui n'est pas n'importe laquelle: «Je jure par Apollon, médecin, par Hygie et Panacée, par tous les dieux et toutes les déesses, les prenant à témoins que je remplirai, suivant mes forces et ma capacité, le serment et l'engagement suivant».

Il est évident que l'invocation cherche un fondement plus solide que notre propre conscience. Pour trouver les principes les plus solides pouvant nous permettre de dépasser le niveau de la subjectivité, le „Serment d'Hippocrate” se réfère à la Réalité divine, au delà de nos opinions quotidiennes. Lauteur du „Serment" cherche un fondement objectif et il se réfère à l'instance suprême des

3 Le «Code d'Hammurabi» est l'un des plus anciens textes juridiques, que nous connaissons. Il fut réalisée sur l'initiative du roi de Babylone Hammurabi en 1750 av. J. C. voir: André Finet: «Le Code de Hammurabi», Le Cerf, 2002. 
hommes. Ainsi la réflexion morale chez Hippocrate trouve son enracinement dans la réalité surnaturelle pour ne pas plonger dans des opinions et des préjugés subjectifs. Elle s'appuie sur la vérité, car seuls les dieux sont capables d'atteindre la vérité absolue 4 .

La deuxième partie du «Serment d'Hippocrate» est consacrée à la responsabilité du développement de la profession, selon une bonne conscience corporative établie sur le principe de la solidarité.

"Je mettrai mon maître de médecine au même rang que les auteurs de mes jours, je partagerai avec lui mon avoir et, le cas échéant, je pourvoirai à ses besoins ; je tiendrai ses enfants pour des frères, et, s'ils désirent apprendre la médecine, je la leur enseignerai sans salaire ni engagement. Je ferai part de mes préceptes, des leçons orales et $d u$ reste de lenseignement à mes fils, à ceux de mon maître et aux disciples liés par engagement et un serment suivant la loi médicale, mais à nul autre».

Ensuite le «Serment d'Hippocrate» désigne l'homme malade comme la seule cible de la médecine vers laquelle le médecin doit tourner son l'engagement personnel et professionnel pour apporter un bon soin aux patients en évitant les sorts dus aux injustices.

L'exigeant morale d'Hippocrate est très concrète, s'agissant de la protection de la vie.

"Je ne remettrai à personne du poison, si on m'en demande, ni ne prendrai l'initiative d'une pareille suggestion; semblablement, je ne remettrai à aucune femme un pessaire abortif».

Cette phrase univoque, obstacle terrible à nos contemporains nus empêche de recevoir le «Serment» en entier. Elle ne nous permet pas une libre interprétation. C’est le clou de la totalité de léthique dans la tradition hippocratique. La vérité ne nous laisse pas délibérer pour nous libérer de notre responsabilité au sujet de la vie de notre prochain. Pour faire face aux exigences imposées par l'»ethos» de la profession, la confiance sociale, il nous faut de la force et une certaine formation professionnelle.

"Je passerai ma vie et jexercerai mon art dans l'innocence et la pureté.»

Cette promesse couvre un secret pour une part déjà oublié : pour bien pratiquer quelque profession que ce soit, il faut d'abord être un homme honnête et droit. Elle est particulièrement actuelle pour ceux à qui l'on confie une certaine responsabilité. Évidemment, cette responsabilité pèse surtout sur les domaines de la médecine à propos de notre santé et de la protection de la vie. Pour agir dans le bon sens, il nous faut appliquer le principe de la compétence. Ainsi Hippocrate propose dans son «Serment»:

4 Les Pythagoriciens affirmaient que la sagesse (la vérité) appartenait aux dieux. Aussi, même les philosophes ne peuvent l'atteindre et restent uniquement les amis de la sagesse. 
"Je ne pratiquerai pas l'opération de la taille, [mais je laisserai cela à ceux qui sén occupent professionnellement $]^{5}$ ».

La partie suivante du texte classique parle de la dignité humaine au-delà des sexes, le statut social. La dignité exige une égalité indépendante de toutes circonstances. Elle nous oblige aussi d'assumer la confiance du patient, de garder son intimité personnelle. Le secret professionnel est un facteur important de l'«ethos» de la profession, de la confiance publique. Garder le secret, c'est respecter l'intégralité de la personne. C'est une règle du comportement de chaque profession.

Chaque phrase du «Serment d'Hippocrate» a son importance irremplaçable. Mais la conclusion nous dévoile le véritable ordre de la morale. On ne parle pas ici de sanctions imposées par une autorité. L’autorité la plus élevée est notre conscience personnelle. Aussi, au cours des siècles, chaque étudiant en médecine, avant d'entrer dans la pratique médicale, faisait la promesse:

"Si je remplis ce serment sans l'enfreindre, qu'il me soit donné de jouir heureusement de la vie et de ma profession, honoré à jamais des hommes; si je le viole et que je me parjure, puisse-je avoir un sort contraire».

La réception du première code d'éthique médicale a trouvé un développement important de lépoque d'Aristote à la scolastique, créant une vraie tradition pour unir la sagesse grecque et la foi chrétienne ${ }^{6}$. La charité chrétienne a enrichi la sagesse d'Hippocrate en créant une belle tradition de la médecine européenne. $\mathrm{Au} \mathrm{XV}$ e siècle, on trouve à l'Université de Cracovie «le Serment d'Hippocrate» enrichi par la foi chrétienne. Cet enrichissement se manifeste dès les premières phrases du Serment où l'on a remplacé les dieux grecs par la Sainte Trinité: «Le Père, Le Fils et le Saint Esprit». Tous les adeptes de la médecine ont prononcé ce serment jusqu'au temps du siècle des Lumières. Dans certains «Serments», on essaie de remplacer l'invocation se référant à la Sainte Trinité par la conscience de l'importance de la profession médicale, en se référant à sa tradition. Selon les modes qui ont changé avec les nouvelles idéologies, on se réfère à l'humanité, soit au socialisme comme l'une des valeurs les plus importantes des pays communistes. ${ }^{7}$ Mais finalement nous nous sommes retrouvés face à un rejet

Selon la traduction basée sur l’édition: Hippocrate: «Opera omnia» vol. 2, Leipzig, 1892.

6 Dans cette tradition on trouve des personnages très importants dans l'histoire de la médecine comme le grand philosophe et médecin perse Avicenne (980-1037) qui représentait le monde musulman et un autre maître du Moyen Age - médecin et philosophes juif d'Égypte Moïse Maïmonides (1138-1204). Sa «Prière» est très souvent citée à côté du «Serment d'Hippocrate».

Aujourd'hui la plupart des «Serments» s'appuient sur le texte de la «Déclaration de Genève» adoptée par la $2^{\text {é }}$ Assemblée Générale de l'Association Médicale Mondiale (OMS) a Genève en Septembre 1948. Danc ce document nous trouvons une autre invocation, par rapport a celle, que nous avons dans le texte d'Hippocrate: «Au moment dêtre admis comme membre de la profession médicale: Je prends l'engagement solennel de consacrer ma vie au service de l'humanité; Je témoi- 
final du «Serment». On ne propose plus le texte originel aux étudiants en disant qu'il n'est plus actuel. Évidemment, à l'époque à laquelle, dans certains pays, l'avortement et l'euthanasie ont acquis un statut légal, le texte sur lequel s'appuie la formation professionnelle des médecins est devenu gênant ${ }^{8}$. Même parmi ceux, qui garde encore les principes du Serment on peut trouver ceux, qui sont prêts a justifier, que le Serment est dépasse, car la médecine a évolue. Celle qui est représente par Hippocrat a un caracter paternaliste. On souligne parfois, avec une raison incontestable, que la relation entre le patient et le médecin exige une activité et responsabilité de deux cotes. Il est vraie, que chez Hippocrate on trouve pas cette juste remarque, mais «Le serment d'Hippocrate» c'est le serment des médecins, pas des patients. Alors peut être en prenant le rôle du patient dans le traitement (y compris la diagnostique, réhabilitation ets) il nous faut un jour préparer un serment pour les patients. Et le «Serment d'Hippocrate» peuvent être un excellent exemples.

\section{Conclusions}

«Le Serment d'Hippocrate» porte une forte conscience de l'unité corporative qui est responsable du niveau moral dans son milieu. Aujourd'hui, on appelle cela «l'ethos de la profession de la confiance publique». Nous le savons bien qu'aujourd'hui ce ne sont pas uniquement les médecins qui représentent «la profession de la confiance publique». La liste des professions s'accroît avec le développement de notre société. La société contemporaine impose des exigences morales aux éducateurs, aux juristes, mais aussi aux politiciens, aux économistes et aux hommes d'affaires. C'est pour cela que nous pouvons poser la question de savoir si Hippocrate peut être un exemple pour les hommes d'affaires. La question est de plus en plus actuelle, avec le développement de la médecine, avec le développement de traitements extrêmement chers, la médecine devenant un vaste problème politique et économique. Il n’est pas uniquement valable pour les pays pauvres ou en voie de développement, mais aussi pour les sociétés développées où la consommation est plus élevé.

La médecine est devenu un facteur important dans le marché libre de la société civile. Ainsi le domaine de la médecine, en prenant une dimension nouvelle, impose de nouvelles exigences liées aux recherches scientifiques, au développement de la technique et au développement de la société qui s’appuie

gnerai à mes maîtres le respect et la reconnaissance qui leur sont dus; J’exercerai ma profession avec conscience et dignité...»

8 Dans le texte de la «Déclaration de Genève» on parle plus ni de l'avortement, ni d'euthanasie. Il est vrai que la phrase: «Je garderai le respect absolu de la vie humaine» et si fort et univoque, qu'il $\mathrm{n} \mathrm{y}$ a rien à ajouter à condition que l'embryon humain soit traité comme une personne humaine, ce qui n’est plus évident pour certains auteurs. 
sur des décisions justes et raisonnables au plan politique et économique. Chaque décision médicale et économique présuppose une anthropologie. Chaque décision cache une vision de l'homme, sa dimension et son destin. Il est donc très important que la médecine et les autres professions de la confiance publique se posent la question à chaque instance : qu'est-ce que l'homme? quel est son destin aux plans biologique et spirituel ?

«Le Serment d'Hippocrate» s'appuie sur des valeurs objectives qui expriment la vérité de la dignité humaine ${ }^{9}$. Grâce à ses références objectives, il reste toujours actuel. La nature humaine ne change pas. Seuls notre conscience et notre regard sur l'homme changent. Le premier commandement «Tu ne tueras point» est toujours actuel et en toutes circonstances. Il est vrai qu'avec le développement de la technique la mort peut être réalisée de manières très différentes, sous la forme de l'avortement, par un acte humanitaire, légal et légitime (selon certaines lois) ou de l'euthanasie.

La civilisation dans toutes ses dimensions a comme but le développement et lépanouissement de la personne humaine. Sinon, elle devient une sorte de prison idéologique qui détruit dans l'homme ce qui lui est le plus précieux. L'homme nest pas uniquement un élément de la société, mais il est toujours un sujet à qui l'on confie certaines responsabilités. On voit bien dans le serment d'Hippocrate qu'au centre de notre activité dans chaque domaine il y a la personne humaine. Tout le reste n'appartient qu'à l'ordre des moyens.

Si Hippocrate avait été un homme d'affaires, il aurait bien pu nous montrer que le développement, les profits, la production, l'organisation et le progrès avaient un sens à condition dêtre liés au bien de la personne humaine.

Il n y a que l'avortement, l'euthanasie qui sont décidément rejette par Hippocrate. Les problèmes sont multiples est beaucoup plus différents. Surtout au niveau économique. Le médecin travail dans un système de la sécurité sociale, l'organisation de la santé publique bien implante dans une politique économique. C'est une de raison pour laquelle tradition de léthique hippocratique reste un instrument toujours actuelle, aussi dans cette large contexte.

Le Serment d'Hippocrate ne parle pas directement des finances qui sont nécessaires pour pratiquer la médecine. Il me semble tout a fait normale. Largent fait partie des moyens, le Serment parle plutôt des principes. Mais les moyens ne peuvent pas utilises raisonnablement sans aucune références aux principes moraux qui garde la personne humaine comme une seul but de notre activité, quelque soit la domaine. Si non, même l'économie perdre sa vocation. Et dans

9 L'histoire de la réception du «Sermont d'Hippocrate» fondée sur la lois naturelle et bien enrichie par la loi révélée au moyen age nous montre cette unite entre deux lois qui sont de plus en plus abondonée et remplacé par la loi positive. Les précisions concernates la loi éternele, loi naturelle, loi révélée, loi positive et droits des gens a découvrir dans l'ouvrage de Partick de Laubier: «La loi naturelle, le politique et la religion» Edition Parole e4t Silece, Langres 2004 p 11 - 14. 
cette perspective «Le Serment d'Hippocrate» qui place la personne du malade au cśur de la médecine, il peut nous donner une bonne orientation pour trouver la réponse aux questionnes suivantes:

- est il économiquement raisonnable d'apporter les traitements parfois très chères aux vieille personnes pour prolonger la vie a tout prix?

- est il économiquement raisonnable soutenir la vie a ceux qui par une déformation prénatale soit par l'accident restent dans létat végétative de sa vie?

- comment composer les dépenses dans le budget nationale pour apporter un service médical aux citoyens? (en Pologne Budget pour 2009 prévoit 4,6 mld sur 288 mld des dépenses)

- est il acceptable que les firmes pharmaceutiques qui sont fort au niveau économique ont une influence importante sur les décisions politiques et même sur les décisions thérapeutiques fait par des médecins?

- quelle sont des limites des informations confidentiel concernant notre santé exigés par des agents d'assurances?

- est il juste, que les sociétés riche ont un accès aux traitements les plus sophistique pour garder la beauté, la forme physique, changer le sexe etc. pendant que dans les pays pauvres des millions des enfants naurons jamais la chance de rencontrer un médecin? ${ }^{10}$

Les répons a ceux questionnes sont a trouver chez Hippocrate. Même si le problèmes soulevé sont tout a fait nouveau. Il suffit seulement de voir d'abord la personne humaine qui est sujet de la médecine et de chaque autre domaine liée a la médecine ${ }^{11}$.

Le système de la sainte publique contienne le monde des producteurs des pharmaceutiques d 'un cote et les monde des médecins et diagnostitiens. Chacun jue son rôle dans le système, mais les relations entre eux ne sont pas toujours le même. Le marche des pharmaceutiques demandent directement des prescripteurs (médecins) et des diagnostitiens. Un seul effort qui peuvent etre intrepri par des entreprisrs pharmaceutiques qui va développer son importance de point de vu de l'économie, c'est de se lancer dans des recherches qui vont perfectionner ses produits. Sinon, ils doivent se lances dans des actions publicitaires, qui sont strictement reglamentées. Alors il reste encore de faire la pression aux médecins, diagnostitiens laboratoires et pourquoi pas sur des patients. Sa peut se réaliser

10 On trouve cette question dramatique à travers des texstes de Raoul Follereau rasamblé en cinque volumes. Voir par example le texte «Ne ditez paz: l'ai faim» dans: «Les oevres completes de Raoul Follereau» présentés et commentées par André Récipon, Editions Fondation Raoul Follereau, Paris 2002, p 81.

11 «Le cpncepte de personne introduit une nouvelle dimension, celle qui fait l'irréductibilité et la spécificité de lêtre humaine en tant q'humaine. Car celui-ci est dit sujet dans un sens nouveau et plus profonde, dans la mesure ou il est capable d'une vie et d'activités qui sont d'ordre spirituel.» Georges Cottier: «Défis éthiques» Editions Saint Augustin, Saint Maurice 1996, p 206. 
a plusieurs manières plus au moins honette. Les cadeaux, les voyages dans les antilles a titre «éducatif» etc. Il faut ajouter l'importance de la presse, qui a travers des compagnies d'information peuvent monte la demande du cote des patients.

Depuis quelques années je travaille dans la domaine de la diagnostique laboratoire et en même temps auprès des producteurs pharmaceutiques présentes au marché polonais. Les deux domaines présentent deux visions de la politique financière. L'un souligne importance de la médecine préventive dans laquelle les examines laboratoires jouent un rôle principale, l'autre - la médecine, qui apporte le soins, qui sauve la santé et même parfois la vie humaine. Les arguments des diagnostitiens sont suivant: la diagnostique préventive est moins chère que le traitement du malade qui exige a la fin une réhabilitation.

Le résultat de la diagnostique s'est prévenir la maladie et laisse personne en bonne sainte, le traitement n'est pas toujours efficace, et le malade reste plus au moins invalide en restant en charge de la société.

La pression du cote des laboratoires pharmaceutiques sur développement sa domaine a aussi ses forts raisons. Il est évident, qu'il faut apporter le soins au malades et il faut faire des recherches les plus avances pour apporter les secours a ceux qui soufrent.

Il est bien qu'il y a les discussions de cet joindre autour de problème de la médecine, mais on a ceux qui disent; il y a trop d'argent en jeu pour soccuper des principes moraux. Le but d'en entrepreneur soit dans la domaine de la diagnostiques ou pharmaceutiques, s'est rentabilité. Il faut pas trop rêver. C'est depuis des siècles, que l'économie se détaché de l'éthique (étroitement lie chez Aristote $)^{12}$ et on peut pas retourner dans le temps. Et ou est Hippocrate?

Malgré que nous sommes les témoins d'abondance des valeurs humaine détruits par des idéologies multiples, a chaque instant que nous sommes oblige de prendre une décision il nous faut se référer au bonnes références. Ceux qui nous apporte une vaste et profond tradition hippocratique nous donne un fondement solide, bien implante dans notre culture judeo-chrétienne.

On peut avoir l'impression que l'homme n'a plus d'importance dans l'univers. Il est important, mais relativement aux conditions de la vie, à la place de la société, etc. La dignité ${ }^{13}$ de la vie humaine comme telle est remplacée par la qualité de la vie ${ }^{14}$.

12 Aristote: «Politique», traduction, introduction, notes et index par J. Tricot IIIe édition, Libraire Philosophique Vrin, Paris 1982 p 24 - 31.

13 On utilise très souvent aujourd'hui na notion «dignité» par exemple dans le «Traite européenne» mais la dignité séparé du notion divinité perd son sens. Littre dit que la dignité, s'est garder dans l'autre ce qui est cher en moi même. Les autres disent, que la dignité vienne de la liberté humaine, et la liberté - d'où elle vienne? Évidement de notre raison, mais les personnes qui n'ont pas la possibilité de s'exprimer pour manifester sa nature raisonnables - ont ils la dignité humaine?

14 L'histoire du XXe siècle nous montre les terribles conséquences qui provoque la la terminologie utilisée par les nazis allemands «lebensunwerte Leben» (la vie qui n’a plus de valeur). Voir: Karl Binding in Alfred Hoche: «Die Freigabe der Vernichtung lebensunwerten Lebens», Laipzich 1920. 
Mais la nature ne peut pas se taire. Et nous voyons une soif de vérité et une soif de vraies valeurs. Saint Augustin disait que la vérité n’est pas méritée, et en même temps on ne trouve personne qui accepte dêtre privé de vérité.

\section{STRESZCZENIE}

Artykuł jest prezentacją wykładu wygłoszonego na Międzynarodowej Sesji Naukowej „Etyka, finanse, odpowiedzialność” odbywającej się w w dniach 3-4 października 2008 w Château de Bossey koło Genewy. Szukając źródeł refleksji etycznej nad trudnymi problemami moralnymi współczesnego świata autor zajął się najstarszym znanym tekstem etycznym ujmującym podstawowe zagadnienia moralne związane $\mathrm{z}$ wykonywaniem zawodu lekarza, jakim jest „Przysięga Hipokratesa”. Przedstawiając bogatą, z gruntu filozoficzno-etyczną treść „Przysięgi” oraz historię budowania się opartej na niej tradycji hipokratejskiej wzbogaconej o doświadczenie antropologii chrześcijańskiej autor identyfikuje podstawowe jej normy i zasady. Niestety, od czasów oświecenia, a szczególnie od dziewiętnastowiecznego pozytywizmu doszło do poważnego zachwiania tej tradycji. Wraz z zakwestionowaniem arystotelesowsko-tomistycznej antropologii pojawiła się wątpliwość dotycząca racji chronienia człowieka od poczęcia, aż do jego naturalnej śmierci. Tymczasem nowe regulacje prawne związane z postępem w naukach medycznych, domagają się głębokiej refleksji z poziomu antropologii filozoficznej, która przywróciłaby na nowo blask zapomnianej, tradycji hipokratejskiej, która w centrum medycznych działań stawia człowieka potrzebującego pomocy.

\section{SUMMARY}

The article is a presentation of a lecture delivered on the International Science Session "Ethics, Finances and Responsibility", which took place in October the $3^{\text {rd }}$ and $4^{\text {th }}, 2008$ in Chateau de Bossey near Geneva. Searching for sources of ethic thoughts connected with difficult moral problems of the contemporary world, the author deals with the oldest, wellknown writing about the ethical practice of medicine, the Hippocratic Oath. Presenting the plenteous, philosophical- ethical contents of the Oath and the history of the growth on it the Hippocratic tradition enriched in experience of the Christian anthropology, the author identifies its norms and rules. Unfortunately, since the Enlightenment times, especially the nineteenth century Positivism the tradition has been seriously disturbed. Together with the questioning the Aristotelean-Thomism anthropology, the doubts appeared related to the matter of protection of life since the conception until the natural death. Whereas new, legal regulations connected with the progress of in medical studies request deep anthropological and philosophical reflection, which would bring back the importance of the forgotten Hippocratic tradition, where in the center of medical actions is a man who needs help. 